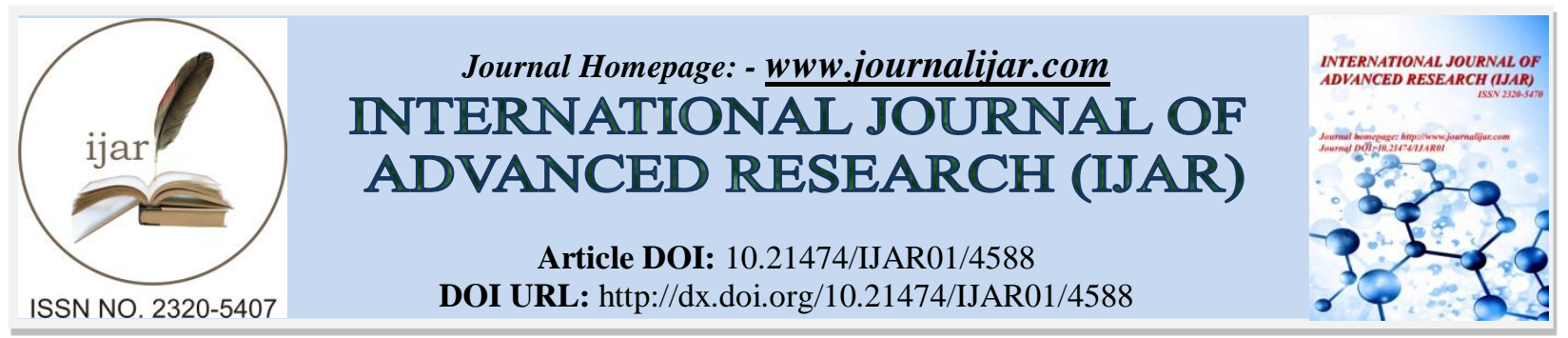

RESEARCH ARTICLE

\title{
SEMI SYMMETRIC NON-METRIC $S$-CONNECTION ON A GENERALIZED CONTACT METRIC STRUCTURE MANIFOLD.
}

Shalini Singh.

Department of Applied Mathematics, JSS Academy of Technical education, Noida, 201301, India.

\section{Manuscript Info}

\section{Manuscript History}

Received: 24 April 2017

Final Accepted: 26 May 2017

Published: June 2017

Key words:-

$C^{\infty}$-manifold, generalized contact metric structure manifold, semi-

symmetric non-metric $S$-connection, curvature tensor.

\section{Abstract}

In the present paper, we define a semi-symmetric non-metric $S$ connection $\tilde{B}$ on a generalized contact metric structure manifold $M_{n}$ and define the curvature tensor of $M_{n}$ with respect to semisymmetric non-metric $S$-connection. It has been shown that if a generalized contact metric structure manifold admits a semisymmetric non-metric $S$-connection whose curvature tensor is locally isometric to the unit sphere $S^{n}(1)$, then the conformal and conharmonic curvature tensor with respect to Riemannian connection are identical iff $n-\frac{a^{2}}{c}(n+2)=0$. Also it has been shown that if a generalized contact metric structure manifold admits a semisymmetric non-metric $S$-connection whose curvature tensor is locally isometric to the unit sphere $S^{n}(1)$, then the con-circular curvature tensor coincides with curvature tensor with respect to the Riemannian connection if $n-\frac{a^{2}}{c}(n+2)=0$. Some other useful results on projective curvature tensor $W$ and con-circular curvature tensor $C$ with respect to semi-symmetric non-metric $S$-connection have been obtained.

Copy Right, IJAR, 2017,. All rights reserved.

\section{Introduction:-}

Consider a differentiable manifold $M_{n}$ of differentiability class $C^{\infty}$. Let there exist in $M_{n}$ a vector valued $C^{\infty}$ linear function $\Phi$, a $C^{\infty}$ - vector field $\eta$ and a $C^{\infty}$-one form $\xi$ such that

$$
\begin{aligned}
& \Phi^{2}(X)=a^{2} X+c \xi(X) \eta \\
& \bar{\eta}=0
\end{aligned}
$$




$$
G(\bar{X}, \bar{Y})=-a^{2} G(X, Y)-c \xi(X) \xi(Y)
$$

Where $\Phi(X)=\bar{X}, a$ is a nonzero complex number and $c$ is an integer, then the set $(\Phi, \eta, a, c, \xi, G)$ satisfying (1.1) to (1.3) is called a generalized contact metric structure and $M_{n}$ equipped with a generalized contact metric structure will be called a generalized contact metric structure manifold.

It is easy to calculate in $M_{n}$

$$
\begin{aligned}
& \xi(\eta)=\frac{a^{2}}{c} \\
& \xi(\bar{X})=0
\end{aligned}
$$

and

$$
G(X, \eta) \underline{\underline{\operatorname{def}}} \xi(X)
$$

Remark 1.1: A generalized contact metric structure manifold $(\Phi, \eta, a, c, \xi, G)$ gives an almost Norden contact metric manifold [5], an almost para norden contact metric manifold, an almost para contact metric manifold [1] or Lorentzian para contact metric manifold $\left(a^{2}=-1, c=1\right),\left(a^{2}=-1, c=-1\right),\left(a^{2}=1, c=1\right)$ or $\left(a^{2}=1, c=-1\right)$

[2] according as

Definition 1.1: A $C^{\infty}$-manifold $M_{n}$, satisfying

$$
D_{X} \eta=\Phi(X) \underline{\underline{\operatorname{def}}} \bar{X}
$$

will be denoted by $M_{n}{ }^{*}$

In $M_{n}{ }^{*}$, we can easily show that

$$
\left(D_{X} \xi\right)(Y)=` \Phi(X, Y)=\left(D_{Y} \xi\right)(X)
$$

where

$$
`(X, Y) \underline{\underline{\operatorname{def}}} G(\bar{X}, Y)=G(X, \bar{Y})=` \Phi(Y, X)
$$

Definition 1.2: An affine connection $\tilde{B}$ is said to be metric if

$$
\tilde{B}_{X} G=0
$$

The affine metric connection $\tilde{B}$ satisfying

$$
\left(\tilde{B}_{X} \Phi\right)(Y)=\xi(Y) X-G(X, Y) \eta
$$

is called metric $S$ - connection.

A metric $S$ - connection $\tilde{B}$ is called semi-symmetric non-metric $S$ - connection if

$$
\tilde{B}_{X} Y=D_{X} Y-\xi(Y) X-G(X, Y) \eta
$$

Where $D$ is the Riemannian connection.

Also

$$
\left(\tilde{B}_{X} G\right)(Y, Z)=2 \xi(Y) G(X, Z)+2 \xi(Z) G(X, Y)
$$

which implies

$$
S(X, Y)=\xi(Y) \bar{X}-\xi(X) \bar{Y}
$$

where $S$ is the torsion tensor of connection $\tilde{B}$.

The curvature tensor with respect to the semi-symmetric non-metric $S$-connection is defined as

$$
\tilde{R}(X, Y, Z) \underline{\underline{\operatorname{def}}} \tilde{B}_{X} \tilde{B}_{Y} Z-\tilde{B}_{Y} \tilde{B}_{X} Z-\tilde{B}_{[X, Y]^{Z}}
$$

Using (1.12) in (1.14), we get 


$$
\begin{aligned}
& \tilde{R}(X, Y, Z)=K(X, Y, Z)-\beta(X, Z) Y+\beta(Y, Z) X-G(Y, Z)\left(D_{X} \eta-\xi(X) \eta\right) \\
& +G(X, Z)\left(D_{Y} \eta-\xi(Y) \eta\right)
\end{aligned}
$$

where

$$
\beta(X, Y)=\left(D_{X} \xi\right)(Y)+\xi(X) \xi(Y)+G(X, Y) \xi(\eta)
$$

and

$$
\left.K(X, Y, Z) \stackrel{\text { def }}{\underline{\underline{n}}} D_{X} D_{Y} Z-D_{Y} D_{X} Z-D_{[X, Y]}\right]^{Z}
$$

where $\tilde{R}$ and $K$ be the curvature tensors with respect to the connection $\tilde{B}$ and $D$ respectively.

Using (1.7) in (1.15), we get

(1.18) $\tilde{R}(X, Y, Z)=K(X, Y, Z)-\beta(X, Z) Y+\beta(Y, Z) X-G(Y, Z)(\bar{X}-\xi(X) \eta)+G(X, Z)(\bar{Y}-\xi(Y) \eta)$

If $\tilde{R}(X, Y, Z)=0$ then above equation becomes

$$
K(X, Y, Z)-\beta(X, Z) Y+\beta(Y, Z) X-G(Y, Z)(\bar{X}-\xi(X) \eta)+G(X, Z)(\bar{Y}-\xi(Y) \eta)=0
$$

Contracting above equation with respect to $X$, we get

$$
\operatorname{Ric}(Y, Z)-\beta(Y, Z)+n \beta(Y, Z)+\frac{a^{2}}{c} G(Y, Z)+G(\bar{Y}, Z)-\xi(Y) \xi(Z)=0
$$

Using (1.16) in (1.20), we get

$$
c \operatorname{Ric}(Y, Z)+c(n-1)\left[\Phi(Y, Z)+\xi(Y) \xi(Z)+\frac{a^{2}}{c} G(Y, Z)\right]+G(\bar{Y}, \bar{Z})+c G(\bar{Y}, Z)=0
$$

Contracting above equation with respect to $Z$, we get

$$
r Y+n\left(\frac{a^{2}}{c} Y+\bar{Y}\right)+(n-2) \xi(Y) \eta=0
$$

Contracting above equation with respect to $Y$, we get

$$
\tilde{R}=-\frac{a^{2}}{c}(n+2)(n-1)
$$

Where Ric and $\tilde{R}$ are Ricci tensor and scalar curvature of the manifold respectively.

The Projective curvature tensor $W$, Con-harmonic curvature tensor $L$, Conformal curvature tensor $V$ and Concircular curvature tensor $C$ in a Riemannian manifold are given by [3], [4].

$$
W(X, Y, Z)=K(X, Y, Z)-\frac{1}{(n-1)}[\operatorname{Ric}(Y, Z) X-\operatorname{Ric}(X, Z) Y]
$$

$$
L(X, Y, Z)=K(X, Y, Z)-\frac{1}{(n-2)}[\operatorname{Ric}(Y, Z) X-\operatorname{Ric}(X, Z) Y+G(Y, Z) r(X)-G(X, Z) r(Y)]
$$$$
V(X, Y, Z)=K(X, Y, Z)-\frac{1}{(n-2)}[\operatorname{Ric}(Y, Z) X-\operatorname{Ric}(X, Z) Y+G(Y, Z) r(X)
$$

$$
-G(X, Z) r(Y)]+\frac{\tilde{R}}{(n-1)(n-2)}[G(Y, Z) X-G(X, Z) Y]
$$

where

(1.28) $W(X, Y, Z, T) \underline{\underline{\operatorname{def}}} G(W(X, Y, Z), T)$ 


$$
\begin{aligned}
& \mathcal{L}(X, Y, Z, T) \underline{\underline{\operatorname{def}}} G(L(X, Y, Z), T) \\
& V(X, Y, Z, T) \underline{\underline{\operatorname{def}}} G(Q(X, Y, Z), T) \\
& C(X, Y, Z, T) \underline{\underline{\operatorname{def}}} G(C(X, Y, Z), T)
\end{aligned}
$$

\section{Curvature Tensors:-}

Theorem 2.1:- If a generalized contact metric structure manifold admits a semi-symmetric non-metric $S$ connection whose curvature tensor is locally isometric to the unit sphere $S^{(n)}(1)$, then the Conformal and Conharmonic curvature tensors with respect to the Riemannian connection are identical iff $n-\frac{a^{2}}{c}(n+2)=0$

Proof: If the curvature tensor with respect to the semi-symmetric non metric $S$ - connection is locally isometric to the unit sphere $S^{(n)}(1)$, then

$$
\tilde{R}(X, Y, Z)=G(Y, Z) X-G(X, Z) Y
$$

Using (2.1) in (1.18), we get

$$
\begin{aligned}
& G(Y, Z) X-G(X, Z) Y=K(X, Y, Z)-\beta(X, Z) Y+\beta(Y, Z) X \\
& -G(Y, Z)(\bar{X}-\xi(X) \eta)+G(X, Z)(\bar{Y}-\xi(Y) \eta)
\end{aligned}
$$

Contracting above with respect to $X$ and using (1.4) and (1.9), we get

$$
c \operatorname{Ric}(Y, Z)=c(n-1)\left[G(Y, Z)-` \Phi(Y, Z)-\xi(Y) \xi(Z)-\frac{a^{2}}{c} G(Y, Z)\right]-G(\bar{Y}, \bar{Z})-c G(\bar{Y}, Z)
$$

Contracting above equation with respect to $Z$, we get

$$
c r Y=-c n(Y-\bar{Y})-(n-2) c \xi(Y) \eta-\left(a^{2} n+c\right) Y
$$

Contracting above equation with respect to $Y$, we get

$$
\tilde{R}=(n-1)\left[n-\frac{a^{2}}{c}(n+2)\right]
$$

Where Ric and $\tilde{R}$ are Ricci tensor and scalar curvature of the manifold respectively.

From (2.5), (1.25) and (1.26), we obtain the necessary part of the theorem. Converse part is obvious from (1.25) and (1.26).

Now, using (1.19) and (1.21) in (1.24), we get

$$
\begin{aligned}
& W(X, Y, Z)=\beta(X, Z) Y-\beta(Y, Z) X+G(Y, Z) \bar{X}-G(Y, Z) \xi(X) \eta \\
& -G(X, Z) \bar{Y}+G(X, Z) \xi(Y) \eta+\xi(Y) \xi(Z) X-\xi(X) \xi(Z) Y \\
& +\frac{n}{(n-1)}[G(\bar{Y}, Z) X-G(\bar{X}, Z) Y]+\frac{1}{c(n-1)}[G(\bar{Y}, \bar{Z}) X-G(\bar{X}, \bar{Z}) Y] \\
& +\frac{a^{2}}{c}[G(X, Z) Y-G(Y, Z) X]
\end{aligned}
$$

Now operating $G$ on both the sides of above equation and using (1.6) and (1.28), we get

$$
\begin{aligned}
& W(X, Y, Z, T)=\beta(X, Z) G(Y, T)-\beta(Y, Z) G(X, T)+G(Y, Z) G(\bar{X}, T) \\
& -G(Y, Z) \xi(X) \xi(T)-G(X, Z) G(\bar{Y}, T)+G(X, Z) \xi(Y) \xi(T)+\xi(Y) \xi(Z) G(X, T) \\
& -\xi(X) \xi(Z) G(Y, T)+\frac{n}{(n-1)}[G(\bar{Y}, Z) G(X, T)-G(\bar{X}, Z) G(Y, T)]
\end{aligned}
$$




$$
+\frac{1}{c(n-1)}[G(\bar{Y}, \bar{Z}) G(X, T)-G(\bar{X}, \bar{Z}) G(Y, T)]+\frac{a^{2}}{c}[G(X, Z) G(Y, T)-G(Y, Z) G(X, T)]
$$

Theorem 2.2: On a $C^{\infty}$-manifold $M_{n}$, we have

$$
\begin{aligned}
& W(X, Y, Z, \eta)=\beta(X, Z) \xi(Y)-\beta(Y, Z) \xi(X)+\frac{n}{(n-1)}[\cdot \Phi(Y, Z) \xi(X)-` \Phi(X, Z) \xi(Y)] \\
& +\frac{1}{c(n-1)}[G(\bar{Y}, \bar{Z}) \xi(X)-G(\bar{X}, \bar{Z}) \xi(Y)] \\
& W(\bar{X}, \bar{Y}, Z, \eta)=0 \\
& W(\eta, Y, Z, \eta)=\beta(\eta, Z) \xi(Y)-\frac{a^{2}}{c} \beta(Y, Z)+\frac{a^{2}}{c}\left(\frac{n}{n-1}\right) G(\bar{Y}, Z)+\frac{a^{2}}{c^{2}}\left(\frac{n}{n-1}\right) G(\bar{Y}, \bar{Z}) \\
& W(X, Y, \eta, \eta)=\beta(X, \eta) \xi(Y)-\beta(Y, \eta) \xi(X) \\
& W(\eta, Y, Z, T)=\beta(\eta, Z) G(Y, T)-\beta(Y, Z) \xi(T)-\xi(Z) G(\bar{Y}, T) \\
& +2 \xi(Z) \xi(Y) \xi(T)-\frac{2 a^{2}}{c} G(Y, T) \xi(Z)+\frac{n}{(n-1)} G(\bar{Y}, Z) \xi(T)-\frac{1}{c(n-1)} G(\bar{Y}, \bar{Z}) \xi(T) \\
& W(\eta, Y, Z, \eta)=\beta(\eta, Z) \xi(Y)-\frac{a^{2}}{c} \beta(Y, Z)+\frac{a^{2}}{c^{2}(n-1)}[n c G(\bar{Y}, Z)+G(\bar{Y}, \bar{Z})]
\end{aligned}
$$

Proof: Replacing $T$ by $\eta$ in (2.7) and using (1.4), (1.5), (1.6) and (1.9) we get (2.8a).

Replacing $X$ by $\bar{X}$ and $Y$ by $\bar{Y}$ in (2.8a) and using (1.5), we get (2.8b).

Replacing $X$ by $\eta$ in (2.8a) and using (1.2), (1.4), (1.5), (1.6) and (1.9) we get (2.8c).

Replacing $Z$ by $\eta$ in (2.8a) and using (1.2), (1.5) and (1.9), we get (2.8d).

Replacing $X$ by $\eta$ in (2.7) and using (1.2), (1.4) and (1.6), we get (2.8e).

Replacing $T$ by $\eta$ in (2.8e) and using (1.4), (1.5) and (1.6), we get (2.8f).

Theorem 2.3: If a generalized contact metric structure manifold admits a semi-symmetric non metric $S$-connection whose curvature tensor is locally isometric to the unit sphere $S^{(n)}(1)$, then the Con-circular curvature tensor coincides with curvature tensor with respect to the Riemannian connection if $n-\frac{a^{2}}{c}(n+2)=0$

Proof: Using (2.5) in (1.24), we get

$$
C(X, Y, Z)=K(X, Y, Z)-\frac{\left[n-\frac{a^{2}}{c}(n+2)\right]}{n}[G(Y, Z) X-G(X, Z) Y]
$$

which is required proves of the theorem.

Now, using (1.19) and (1.23) in (1.27), we get

$$
\begin{aligned}
& C(X, Y, Z)=\beta(X, Z) Y-\beta(Y, Z) X+G(Y, Z)(\bar{X})-G(Y, Z) \xi(X) \eta \\
& -G(X, Z) \bar{Y}+G(X, Z) \xi(Y) \eta+\frac{a^{2}}{c}\left(\frac{n+2}{n}\right)[G(Y, Z) X-G(X, Z) Y]
\end{aligned}
$$

Operating $G$ on both sides of above equation and using (1.6), (1.9) and (1.31), we get (2.11)

$$
{ }^{`} C(X, Y, Z, T)=\beta(X, Z) G(Y, T)-\beta(Y, Z) G(X, T)+G(Y, Z) \cdot \Phi(X, T)
$$




$$
\begin{aligned}
& -G(Y, Z) \xi(X) \xi(T)-G(X, Z) \cdot \Phi(Y, T)+G(X, Z) \xi(Y) \xi(T) \\
& +\frac{a^{2}}{c}\left(\frac{n+2}{n}\right)[G(Y, Z) G(X, T)-G(X, Z) G(Y, T)]
\end{aligned}
$$

Theorem 2.4:- On $C^{\infty}$-manifold we have

$$
\begin{gathered}
C(\eta, Y, Z, T)=\beta(\eta, Z) G(Y, T)-\beta(Y, Z) \xi(T)-\frac{a^{2}}{c} G(Y, Z) \xi(T) \\
-\xi(Z) G(\bar{Y}, T)+\xi(Y) \xi(Z) \xi(T)+\frac{a^{2}}{c}\left(\frac{n+2}{n}\right)[G(Y, Z) \xi(T)-G(Y, T) \xi(Z)] \\
(2.12 \mathrm{~b}) \quad C(X, Y, Z, \eta)=\beta(X, Z) \xi(Y)-\beta(Y, Z) \xi(X)+\frac{a^{2}}{c} \frac{(n+2)}{n}[G(Y, Z) \xi(X)-G(X, Z) \xi(Y)] \\
-\frac{a^{2}}{c} G(Y, Z) \xi(X)+\frac{a^{2}}{c} G(X, Z) \xi(Y) \\
\text { (2.12c) } C(\eta, Y, Z, \eta)=\beta(\eta, Z) \xi(Y)-\frac{a^{2}}{c} \beta(Y, Z)+\frac{a^{2}}{c} \xi(Y) \xi(X)+\frac{a^{2}}{c} \frac{(n+2)}{n}\left[\frac{a^{2}}{c} G(Y, Z)-\xi(Y) \xi(Z)\right] \\
\text { (2.12d) } \quad C(X, Y, \eta, \eta)=\beta(X, \eta) \xi(Y)-\beta(Y, \eta) \xi(X) \\
\text { (2.12f) } \quad C(\eta, Y, \bar{Z}, \bar{T})=\beta(\eta, \bar{Z}) G(Y, \bar{T}) \\
C(\bar{X}, \bar{Y}, Z, \eta)=0
\end{gathered}
$$

Proof:-Replacing $X$ by $\eta$ in (2.11) and using (1.2), (1.4), (1.5), (1.6) and (1.9), we get (2.12a)

Replacing $T$ by $\eta$ in (2.8) and using (1.4), (1.5), (1.6) and (1.9), we get (2.12b).

Replacing $T$ by $\eta$ in (2.12a) and using (1.4) and (1.6), we get (2.12c).

Replacing $Z$ by $\eta$ in (2.12b) and using (1.6), we get (2.12d).

Replacing $Z$ by $\bar{Z}$ and $T$ by $\bar{T}$ in (2.12a) and using (1.4), we get (2.12e).

Replacing $X$ by $\bar{X}$ and $Y$ by $\bar{Y}$ in (2.12b) and using (1.4), we get (2.12f).

\section{References:-}

1. Adati T. and Matsumoto K. (1977), On almost paracontact Riemannian manifold, TRU maths. 13(2), pp. 22-39.

2. Matsumoto K. (1989), On Lorentzian paracontact manifold, Bull. Yamogata Univ. Nat. Sci., 12, pp. 151-156.

3. Mishra R. S. (1984), Structure on a differentiable manifold and their applications, Chandrama prakashan, Allahabad, India.

4. Mishra R. S. (1995), A course in tensors with applications to Riemannian geometry, Pothishala private Limited, Allahabad, $4^{\text {th }}$ edition.

5. Singh S. D. and Singh D. (1997), Tensor of the type (0,4) in an almost norden contact metric manifold, Acta Cincia Indica, India, $18 \mathrm{M}(1)$, pp. 11-16. 